\title{
Clinical Characteristics and Prognostic Factors of HIV-Positive Patients With Lymphoma in the Poverty Region of China: A Real-World Multicenter Analysis
}

Jinxin Zhang

Southern Medical University Nanfang Hospital

Zhiman Xie

Guangxi Medical University Third Affiliated Hospital: Nanning Second Peoples Hospital

Shanfang Qin

Liuzhou Municipal Liutie Central Hospital

Guangjing Ruan

Nanning Second Peoples Hospital

Shaohang Cai

Southern Medical University Nanfang Hospital

Aili Lu

Southern Medical University Nanfang Hospital

Yihua Wu

Southern Medical University Nanfang Hospital

Juanjuan Chen ( $\sim$ chenjj@smu.edu.cn )

Southern Medical University Nanfang Hospital

Jie Peng

Southern Medical University Nanfang Hospital

\section{Research Article}

Keywords: human immunodefi ciency virus, lymphoma, clinical characteristics, prognostic factor, low income and middle income countries

Posted Date: August 26th, 2021

DOI: https://doi.org/10.21203/rs.3.rs-829347/v1

License: (1) (1) This work is licensed under a Creative Commons Attribution 4.0 International License.

Read Full License 
Clinical characteristics and prognostic factors of HIV-positive patients with lymphoma in the poverty region of China: A real-world multicenter analysis

Jinxin Zhang ${ }^{1,4+}$, Zhiman Xie ${ }^{2+}$, Shanfang Qin $^{3}$, Guangjing Ruan ${ }^{2}$, Shaohang Cai ${ }^{1}$, Aili $\mathrm{Lu}^{1}$, Yihua $\mathrm{Wu}^{2}$, Juanjuan Chen ${ }^{1}$, Jie Peng ${ }^{1 *}$

${ }^{1}$ State Key Laboratory of Organ Failure Research, Guangdong Provincial Key Laboratory of Viral Hepatitis Research, Department of Infectious Diseases, Nanfang Hospital, Southern Medical University, Guangzhou, China. ${ }^{2}$ Department of Infectious Diseases, the Fourth Hospital of Nanning, Nanning, China. ${ }^{3}$ Guangxi AIDS Diagnosis and Treatment Quality Control Center, Longtan Hospital of Guangxi Zhuang Autonomous Region, Liuzhou, China. ${ }^{4}$ Department of Respiratory medicine , University of Chinese Academy of Sciences Shenzhen Hospital.

*Correspondence: Dr. Jie Peng, Email: pjie138@163.com; Dr. Juanjuan Chen, Email: chenjj@smu.edu.cn.

${ }^{+}$Jinxin Zhang and Zhiman Xie have contributed equally to this study. 


\begin{abstract}
Background: People living with HIV (PLWHIV) are at increased risk of Hodgkin and non-Hodgkin lymphoma compared with HIV-negative individuals. However, high-quality epidemiological data for lymphoma among PLWHIV from low-income and middle-income countries are scarce, especially in South China. This retrospective study aimed to better define the clinical characteristics, outcomes, and prognostic factors of $\mathrm{HIV}$-associated lymphoma patients in Guangxi and Guangdong provinces of China in the highly active antiretroviral therapy (HAART) era.
\end{abstract}

Methods: We performed a pooled analysis from existing databases of 143 patients with HIV-positive lymphoma between January 2013 and June 2020 from three hospitals in South China. Survival curves were estimated using the Kaplan-Meier method and compared using the log-rank test. Univariable and multivariable Cox proportional hazard models were performed to identify the association of patient-, lymphoma- and HIV-specific variables with the outcomes progression-free survival (PFS) and overall survival (OS).

Results: Of all 143 HIV-positive lymphoma patients, diffuse large B cell lymphoma (DLBCL) was the most common subtype identified (81.8\%). The median age was 49 years (IQR 18-79), and 118 patients (82.5\%) were male. The mean CD4 T-cell count was 185.5 cells/ $\mu \mathrm{L}$ (IQR 3-1089), and 86 (60.1\%) patients were receiving HAART at enrollment. Opportunistic infections (OIs) were reported in $40(28.0 \%)$ patients. Seventy-seven (53.8\%) patients had stage I/II lymphoma, and the Eastern Cooperative Oncology Group performance status was 0 or 1 in $87(60.8 \%)$ patients. Information on elevated lactate dehydrogenase (LDH) and positive CD20 was available for $72 \%$ $(103 / 143)$ and $96.0 \%$ (48/50), respectively. A total of $65.8 \%(77 / 117)$ of DLBCL patients received $\mathrm{EPOCH}$ - (dose-adjusted etoposide, vincristine, cyclophosphamide, prednisone, and doxorubicin) or CHOP-based (cyclophosphamide, doxorubicin, vincristine, and prednisone) regimens. The 1-year PFS and 1-year OS were $32.98 \%$ and $43.62 \%$ in non-Hodgkin's lymphoma (NHL) patients and $32.18 \%$ and $41.40 \%$ in DLBCL patients, respectively. Advanced age and elevated LDH were significant 
predictors of the outcome in both HIV-associated NHL and DLBCL, and the influence of other factors waxed and waned. A lower CD4/CD8 ratio was an independent poor predictor for 1-year OS in HIV-associated NHL but not for DLBCL.

Conclusions: In our population, HIV-positive patients with lymphoma presented aggressive characteristics and exhibited poor survival outcomes, even in the modern HAART era. Effective HIV-directed therapies reduced the impact of HIV-associated prognostic factors on outcomes; however, lymphoma-related factors, such as age and $\mathrm{LDH}$, were found to be key independent predictors for HIV-associated lymphoma.

Keywords: human immunodeficiency virus, lymphoma, clinical characteristics, prognostic factor, low-income and middle-income countries

\section{Background}

Lymphomas remain a leading cause of cancer morbidity and mortality for people living with HIV (PLWHIV), even in the era of highly active antiretroviral therapy (HAART) [1]. Marked regional differences in population-level HIV control and exposure to carcinogenic cofactors among PLWHIV persist worldwide [2]. In high-income countries, demographic changes among PLWHIV, such as aging and increased life expectancy, might lead to an increased burden of Hodgkin lymphoma (HL) in this population [2]. In contrast, classical AIDS-defining cancers (e.g., Kaposi's sarcoma, aggressive B-cell lymphomas, and invasive cervical cancer) remain the most common malignancies for PLWHIV in low-income and middle-income countries (LMICs) [3]. Notably, non-Hodgkin's lymphoma (NHL) comprises more than 50\% of all AIDS-defining cancers, and approximately 70\%-90\% of AIDS-related lymphomas (ARLs) are high-grade B-cell lymphomas, such as diffuse large B cell lymphoma (DLBCL) and Burkitt's lymphoma (BL) [4, 5]. However, high-quality epidemiological data for lymphoma among PLWHIV from LMICs are scarce and remain an important challenge because of suboptimal population-based cancer registration and diagnostic infrastructure [6].

There is clear geographical disparity in HIV/AIDS among all provincial regions in 
China. HIV/AIDs occurs frequently in the southwestern region (e.g., Guangxi, Yunnan, Guizhou, Sichuan, and Chongqing), the northwestern region of Xinjiang, and in a central province of Henan [7]. In the last decade, a few region-specific, population-level studies have sporadically described the epidemiological features, clinical characteristics, pathobiology, management, or prognosis of HIV-associated lymphomas in China [8-12]. The outcomes of Chinese ARL patients have improved in recent years, especially in high-income regions. However, ARL data on clinical incidence, risk factors, and outcomes in Guangxi and Guangdong provinces of South China are still limited [13].

In this paper, we retrospectively analyzed the clinical characteristics, treatment, outcomes, and predictors of lymphomas in PLWHIV from 3 hospitals in Guangxi and Guangdong provinces.

\section{Methods}

\section{Ethical considerations}

The retrospective study was performed in accordance with the Declaration of Helsinki and was approved by the Institutional Ethics Committee of Nanfang Hospital (study identifier NFEC-2021-178). The committee decided to waive the need for written informed consent from the participants in the study because the data were analyzed retrospectively and anonymously.

\section{Patients and data collection}

This retrospective multicenter study was conducted at Nanfang Hospital Affiliated with Southern Medical University, Fourth Hospital of Nanning, and Longtan Hospital of Guangxi Zhuang Autonomous Region. A total of 143 hospitalized newly diagnosed HIV-positive lymphoma patients from January 2013 to June 2020 were included in the present study. The pathological diagnosis of lymphoma was based on the 2008 World Health Organization (WHO) classification. 
The clinical variables were evaluated, including date of enrollment, age, sex, history of HIV/AIDS, baseline CD4 T-cell count, CD4/CD8 ratio, HIV viral load, histological subtype, Ann Arbor staging classification, Lugano classification, Eastern Cooperative Oncology Group performance status (ECOG PS), number and sites of extranodal sites, tumor size, lactate dehydrogenase (LDH), International Prognosis Index (IPI) score, B symptoms, presence of bone marrow (BM) involvement, type of chemotherapy, use of rituximab, intrathecal injection, concurrent use of HAART with chemotherapy, sexual behavior, illicit drug use, opportunistic infection, nonopportunistic infection, serological hepatitis $\mathrm{B}$ virus (HBV) and hepatitis $\mathrm{C}$ virus (HCV) markers, syphilis, complete blood cell count (CBC), lymphocytes/monocytes ratio (LMR), red cell distribution width (RDW), albumin (ALB), and erythrocyte sedimentation rate (ESR).

\section{Clinical assessments}

Computed tomography (CT) or 18F-fluorodeoxyglucose positron emission tomography/computed tomography (FDG PET/CT) was performed for radiological evaluation. Brain magnetic resonance imaging (MRI) was used to assess CNS involvement. Cheson 2007 criteria or standard Lugano 2014 criteria were used to define complete response (CR), partial response (PR), stable disease (SD), and progressive disease (PD). Progression-free survival (PFS) was defined as the time from diagnosis until progression, relapse or death of any cause. Overall survival (OS) was defined as the time from diagnosis until death of any cause. The major endpoints of this study were PFS and OS.

\section{Statistical analysis}

Survival curves were estimated using the Kaplan-Meier method and compared using the log-rank test. The area under the receiver operating characteristic curve (AUROC) was calculated to determine cutoff values for differentiation between the groups. Variables that were significant in the univariable analyses $(\mathrm{p}<0.05)$ were included in weighted multivariable Cox proportional hazard models. Hazard ratios (HRs) and 95\% 
confidence intervals (CIs) were used to summarize the association between variables and survival. All statistical analyses were performed using the Statistical Package for the Social Sciences (SPSS) version 17.

\section{Results}

\section{Patients' clinical characteristics}

In total, 143 HIV-positive patients with lymphoma, including 139 patients (97.2\%) with NHL and 4 patients $(2.8 \%)$ with HL, from 3 registries during the 7.5 -year period between January 2013 and June 2020 were included. Of those, 131 (91.6\%) patients had AIDS-related lymphoma (ARL), and 12 (8.4\%) had non-ARL (Fig. 1). The most common histologies were DLBCL (81.8\%) and BL (7.0\%). They were followed by PBL $(0.7 \%)$, ARL, not further classified lymphoma (2.1\%), NK/T-cell lymphoma (NKTL, 2.1\%), small lymphocytic lymphoma (SLL, 0.7\%), anaplastic large cell lymphoma (ALCL, 0.7\%), HL (2.8\%), and other non-ARLs (2.1\%) (Fig. 1).

All the demographic data and baseline characteristics are depicted in Table 1. The median age at diagnosis for the entire cohort was 49 (range 18-79) years with $33.6 \%$ of patients older than age 55. Most patients were male $(82.5 \%)$, with a male to female ratio of 4.72:1. The mean CD4 T-cell count (cells/ $\mu \mathrm{L}$ ) was $185.5 \pm 169.446$. Over half of the patients had an ECOG PS $<2(60.8 \%)$ and stage I/II disease (53.8\%). Extranodal involvement in $>1$ site was documented in $25.9 \%$ of patients. LDH was elevated in $72 \%$ of patients, with $33.5 \%$ of patients having LDH measures more than 3 times the upper limit of normal (ULN). According to the IPI, $35.7 \%$ of the patients were low-risk, $26.5 \%$ of the patients were low-intermediate risk, $16.8 \%$ of the patients were high-intermediate risk, and $16.8 \%$ of the patients were high risk.

Almost half of the patients (47.5\%) had sexual behavior, and 4.2\% (6/143) reported the use of an illicit drug in their lifetime. Of the patients, $10.5 \%(15 / 143)$ had hepatitis B virus (HBV) infection, 6.3\% (9/143) had hepatitis C virus (HCV) seroprevalence, and $12.6 \%(18 / 143)$ had syphilis-seropositivity.

\section{Baseline infections}


Almost 79.0\% (113/143) of patients possessed infections, opportunistic or nonopportunistic, at the time of lymphoma diagnosis (Supplementary Table 1). The incidence rates of opportunistic infection (OI) and nonopportunistic events were found to be $28.0 \%(40 / 143)$ and $72.0 \%$ (103/143), respectively. Thirty patients in these populations had OI and non-OI simultaneously. Candida infection was the most common complaint seen in 18.9\% (27/143) of all patients, followed by Pneumocystis jirovecii pneumonia (PJP, 7.0\%, 10/143), cytomegalovirus (CMV) disease (3.5\%, $5 / 143)$, herpes zoster infection $(2.1 \%, 3 / 143)$, mycobacterial infection $(2.1 \%, 3 / 143)$, and Cryptococcus neoformans infection $(0.7 \%, 1 / 143)$. Overall, 7 patients had two OIs and 1 patient had three OIs concurrently (Supplementary Table 1). By univariate analysis of 95 details available for HIV-positive lymphoma patients, opportunistic infection did not predict 1-year OS (Supplementary Figure 1).

\section{HIV treatment}

HAART drugs were obtained from a special outpatient department. Because of poor patient compliance, $16.8 \%$ (24/143) of patients did not receive HAART, and only $60.1 \%$ $(86 / 143)$ of patients were confirmed to receive HAART treatment (Supplementary Table 2). The message of $23.1 \%$ (33/143) patients was undocumented. Most of them chose free pharmaceuticals with TDF/3TC/EFV $(68.6 \%, 59 / 86)$, and only 6 patients $(7.0 \%, 6 / 86)$ included integrase inhibitor-based therapy.

\section{Lymphoma treatment}

The number of patients with other histologies was too few to draw meaningful conclusions regarding clinical outcomes, except DLBCL. Of the 84 patients with AIDS-related DLBCL, details were available for 77 cases, and 7 cases were administered without chemotherapy. These 77 patients received EPOCH(dose-adjusted etoposide, vincristine, cyclophosphamide, prednisone, and doxorubicin) or CHOP-based (cyclophosphamide, doxorubicin, vincristine, and prednisone) regimens with a median cycle of 3 (Supplementary Table 3). The overall response rate (ORR) was $24.7 \%$ after first-line treatment, including $11(14.3 \%)$ patients with 
complete remission (CR) and $8(10.4 \%)$ patients with PR. Among the 50 DLBCL patients with information on CD20 status, CD20 was positive in 96.0\% (48/50) of patients. However, only 6 patients $(7.8 \%, 6 / 77)$ received rituximab-containing regimens with a CR rate of $83.3 \%$ (data not shown). Notably, patients diagnosed before 2019 were less frequently treated with rituximab than those diagnosed from 2019 onwards (5.8\% vs. $25 \%)$. Otherwise, 20 (26.0\%) patients experienced a stable disease (SD), $18(23.3 \%)$ patients experienced a progressive disease (PD), and 20 (26.0\%) patients died. Patient characteristics with subgroups for treatments and outcomes are listed in Supplementary Table 3.

\section{Prognostic factors in NHL}

Details were available for 94 patients with HIV/AIDS-associated NHL, including DLBCL, BL, PBL, ALCL, and NKTL. At the time of analysis, the 1-year PFS and OS in HIV-positive NHL patients were $32.98 \%$ and $43.62 \%$, respectively (Fig. 2A). The 2-year lymphoma-specific PFS and OS were $30.77 \%$ and $35.39 \%$, respectively, from the 66 available patients (Fig. 2B). The significant prognostic factors for 1-year PFS and OS are listed in Table 2 with univariate survival analysis. The optimal cutoff values were 55 years old (age), 160×10 $/ \mathrm{L}$ (CD4 T-cell count), 0.39 (CD4/CD8 ratio),


(LYM, lymphocyte), $2.25 \times 10^{9} / \mathrm{L}$ (LMR), and $35 \mathrm{~g} / \mathrm{L}$ (ALB). Univariate Cox proportional analysis identified several prognostic factors associated with lower 1-year PFS and OS, such as advanced age, worse CD4 T-cell count, lower CD4/CD8 ratio, elevated LDH, higher RDW ratio, increased ESR, reduced LYM, higher IPI score, and hypoalbuminemia (Table 2 and Supplementary Figure 2). The multivariate analysis indicated that advanced age, lower CD4/CD8 ratio, and elevated LDH were independent poor predictors for 1-year OS in HIV/AIDS-related NHL.

\section{Prognostic factors in DLBCL}

The number of cases with another histological subtype was too low to draw meaningful survival outcomes individually, so we investigated the 1-year PFS and OS 
comparison in the 87 HIV-defined DLBCL patients, resulting in $32.18 \%$ and $41.40 \%$, respectively (Fig. 3A). We evaluated the baseline factors for the prognostic value with the same cutoff values of NHL. Age, LDH, RDW ratio, IPI score, and ESR were associated with lower 1-year PFS and OS in the univariate Cox proportional analysis. Otherwise, the CD4 T-cell count, CD4/CD8 ratio, LYM, and albumin were significantly associated with better 1-year PFS and OS (Table 3 and Supplementary Fig. 3). Fig. 3B displays the ROC curve for 1-year OS for a prediction model. These factors were analyzed in a multivariate Cox proportional hazards model with backward elimination. Advanced age and elevated LDH were independent poor predictors for 1-year OS in AIDS-related DLBCL.

\section{Discussion}

PLWHIV experience an increased risk of NHL of approximately 1 in 25 compared with HIV-negative individuals [14]. In the combination HAART era, the epidemiology of HIV/AIDS-associated lymphoma is changing. However, there is a paucity of data on the epidemiology and management of ARL in South China. In the current study, the median age at presentation was 49 years for the entire cohort, which is comparable to that of the global HIV-positive population $[15,16]$ but low compared to the general population of 52.5 years $[2,16]$. The male to female ratio of 4.72:1, with a male predominance in our study, might be an actual underlying biological difference depending on gender. Similar to what has been described in AIDS-defining lymphomas, there were large proportions of high-grade B-cell lymphomas (DLBCL and BL) [1], elevated LDH, extranodal involvement, and stages III or IV in our cohort, which resulted in a high IPI score and a poor prognosis. Most notably, the percentage of DLBCL in our study was higher than that in a previous study, which might be explained by the lack of pathological infrastructure and misdiagnosis in resource-constrained health systems in China. Almost $57.3 \%$ of patients were diagnosed with HIV and lymphoma simultaneously, with a poor median CD4 count. Late diagnosis also explains the high proportion of infections at lymphoma diagnosis. However, infection was not an independent factor for the prediction of death in our 
present study, relying on the improvement of diagnosis and treatment in recent decades. These clinical characteristics are comparable to many international reports on PLWHIV $[2,17]$.

Concomitant HAART was associated with improved CR rates and a trend toward improved OS in AIDS-defined lymphoma [18]. Our data also support the concurrent use of HAART (60.1\%), which benefits from the UNAIDS 95-95-95 worldwide campaign [19]. However, the 1- and 2-year PFS and OS in our study were lower than those reported previously [20-23]. This may be related to the paucity of diagnostic resources, treatment experience, financial woes, and awareness. First, most of the patient population in the present study was of low economic status and received an antiretroviral (ARV) regimen under a variety of schemes funded by the central government. Thus, two nucleoside reverse transcriptase inhibitors (NRTIs) in combination with a nonnucleoside reverse transcriptase inhibitor (NNRTI) or a protease inhibitor (PI) are common profiles, resulting in a low resistance barrier, poor immune reconstitution, and increased risk for drug-drug interactions (DDIs) during polypharmacy chemotherapy. Additionally, low popularity and awareness of integrase strand transfer inhibitors (INSTIs) in Chinese infectious disease hospitals might be another dilemma. Second, another challenge was the simultaneous existence of a high proportion of CD20-positive but poor popularity of rituximab in AIDS-related DLBCL, partly owing to economic constraints. The rituximab-based chemotherapy regimen is an integral management of immunocompetent and immunodeficient CD20-positive lymphoma patients and is associated with improved CR, PFS, and OS rates $[18,20,21,24,25]$. Third, a shorter median treatment duration (less than 3 cycles) and nonstandard regimen (insufficient dose or prolonged chemotherapy interval) resulted in compromising therapeutic efficacy, partly due to poor health care professionalism, patient compliance, and economic conditions in our samples. A total of $65.8 \%(77 / 117)$ of patients with AIDS-related DLBCL received less than 6 cycles of chemotherapy. Fortunately, this situation has improved since 2018, and $39.3 \%$ of AIDS-defining DLBCL patients receive curative-intent treatment with six to eight 
cycles of CHOP- or EPOCH-based strategies. In fact, the latest 2-year OS and PFS rates were $62.7 \%-79.9 \%$ and $56.4 \%-64 \%$ in high-income regions of China, such as Beijing, Shanghai, and Nanjing [8, 10, 12]. All this underscores the necessity to improve the standard hierarchical management and supportive care of HIV/AIDS-associated lymphomas in low-income and middle-income regions of China.

Herein, age and LDH were effective independent prognostic factors for both NHL and DLBCL in the HIV population while the CD4/CD8 ratio was associated with a survival benefit for ARL but not for DLBCL. These two simplified predictors may be more easily implementable in LMICs, given the reproducibility and bedside availability. We did not find low CD4 count, coinfections, high IPI, poor ECOG PS, or advanced stage disease to be independent predictors of mortality, as others have reported [26]. In our study, $60.1 \%(86 / 143)$ of patients were on HAART at the time of lymphoma diagnosis, while in $19.6 \%$ (28/143) of patients, lymphoma was the presentation of underlying HIV. Individual HIV-related factors such as low CD4 count were no longer associated with poor outcomes, even in the era of non-INSTIs from contemporary LMICs [26]. The lack of significance of IPI in the multivariate analysis was somewhat surprising, but it was considered that effective HIV-directed therapies reduced the impact of HIV-related prognostic factors on outcomes [26]. The influence of other factors waxed and waned in the HAART era. Thus, a comparison of IPI, R-IPI (revised IPI), aaIPI (age-adjusted IPI), and NCCN-IPI (National Comprehensive Cancer Network IPI) in AIDS-related DLBCL is needed to discriminate the most important end point (OS) [27].

Improved insights into the biological heterogeneity in the HIV-negative DLBCL population have led to a refinement of disease classification and the development of new therapeutic approaches over the past two decades [28]. However, research on the pathological features and molecular classifications of ARL patients is seriously inadequate. Further study is needed to better understand the phenotypic and genetic heterogeneity of AIDS-associated DLBCL. 
This study has some limitations. First, although our sample is larger than some other literature from China, we report a high proportion $(34.3 \%, 49 / 143)$ of patients lost to follow-up, which could generate some bias in the results, notably regarding mortality data. Second, most patients in our sample were from resource-poor regions in the habit of heterosexually transmitted infections, not among men who have sex with men (MSM). Third, we could not ascertain causality in the association between some variables and death due to high proportions of substandard treatment of lymphoma and infrastructure limitations.

In conclusion, we reported important real-world data on the clinical characteristics, outcomes, and prognostic factors of lymphomas in PLWHIV patients in South China. We also found advanced age and elevated LDH to be key independent predictors for HIV/AIDS-related NHL and DLBCL. Despite poor survival, the outcomes have improved dramatically, and care has progressed sufficiently in the setting of HIV infection from resource-rich countries and regions. Closing this gap is an essential step in decreasing mortality from HIV/AIDS-related malignancies worldwide.

\section{Supplementary Material}

The online version contains supplementary material available at.

\section{Acknowledgements}

Not applicable.

\section{Authors' Contributions}

Jie Peng and Juanjuan Chen carried out the experimental design, participated in the data interpretation and analysis. Jinxin Zhang, Guangjing Ruan, Shanfang Qin, and Yihua $\mathrm{Wu}$ participated in the collection of raw data. Jinxin Zhang, Zhiman Xie, Juanjuan Chen, and Shaohang Cai participated in the data review, analysis, and statistical analysis. Jinxin Zhang and Juanjuan Chen participated in the drafted the manuscripts. Jie Peng, Juanjuan Chen, and Aili Lu participated in the correction of the manuscripts. All authors read and approved the final manuscript. 


\section{Funding}

The authors would like to acknowledge the patients, families, and caregivers who participated in each of these studies. This work was supported by the grants from the Major Science and Technology Special Project of China (2017ZX10302201004008), the National Natural Science Foundation of China (81971949), Natural Science Foundation of Guangdong Province (2018A030313571), the Clinical Research Startup Program of Southern Medical University by High-level University Construction Funding of Guangdong Provincial Department of Education (LC2016PY003), and Major Science and Technology Special Project of Nanning (20193008).

\section{Availability of data and materials}

The data are presented in the manuscript.

\section{Declarations}

\section{Ethics approval and consent to participate}

Not applicable, because this is a review study.

\section{Consent for publication}

Not applicable.

\section{Competing interests}

The authors declare no competing interest. 


\section{References}

1. Noy A. Optimizing treatment of HIV-associated lymphoma. Blood. 2019;134(17):1385-94.

2. Kimani SM, Painschab MS, Horner MJ, Muchengeti M, Fedoriw Y, Shiels MS, et al. Epidemiology of haematological malignancies in people living with HIV. Lancet HIV. 2020;7(9):e641-51.

3. Yarchoan R, Uldrick TS. HIV-Associated Cancers and Related Diseases. N Engl J Med. 2018;378(11):1029-41.

4. Dolcetti R, Giagulli C, He W, Selleri M, Caccuri F, Eyzaguirre LM, et al. Role of HIV-1 matrix protein p17 variants in lymphoma pathogenesis. Proc Natl Acad Sci U S A. $2015 ; 112(46): 14331-6$.

5. Dal Maso L, Franceschi S. Epidemiology of non-Hodgkin lymphomas and other haemolymphopoietic neoplasms in people with AIDS. Lancet Oncol. 2003;4(2):110 9.

6. Horner MJ, Chasimpha S, Spoerri A, Edwards J, Bohlius J, Tweya H, et al. High Cancer Burden Among Antiretroviral Therapy Users in Malawi: A Record Linkage Study of Observational Human Immunodeficiency Virus Cohorts and Cancer Registry Data. Clin Infect Dis. 2019;69(5):829-35.

7. Xu B, Li J, Wang M. Epidemiological and time series analysis on the incidence and death of AIDS and HIV in China. BMC Public Health. 2020;20(1):1906.

8. Sun JJ, Liu L, Wang JR, Shen YZ, Qi TK, Wang ZY, et al. Clinical outcomes of people living with human immunodeficiency virus (HIV) with diffuse large B-cell lymphoma (DLBCL) in Shanghai, China. Chin Med J (Engl). 2020;133(23):2796802.

9. Wang J, Liang R, Hao C, Liu X, Zhang N, Duan X, et al. Survival outcomes of primary cutaneous T-cell lymphoma in HIV-infected patients: a national population-based study. J Investig Med. 2018;66(4):762-7. 
10. Wu J, Miao Y, Qian C, Tao P, Wang X, Dong X, et al. Clinical characteristics and outcomes in HIV-associated diffuse large B-cell lymphoma in China: A retrospective single-center study. J Cancer. 2021;12(10):2903-11.

11. Miao L, Guo N, Feng Y, Rao H, Wang F, Huang Q. High incidence of MYC rearrangement in human immunodeficiency virus-positive plasmablastic lymphoma. Histopathology. 2020;76(2):201-11.

12. Zeng J, Zhang $\mathrm{X}$, Jia L, Wu Y, Tian Y, Zhang Y. Pretreatment lymphocyte-to-monocyte ratios predict AIDS-related diffuse large B-cell lymphoma overall survival. J Med Virol. 2021;93(6):3907-14.

13. Schommers P, Gillor D, Hentrich M, Wyen C, Wolf T, Oette M, et al. Incidence and risk factors for relapses in HIV-associated non-Hodgkin lymphoma as observed in the German HIV-related lymphoma cohort study. Haematologica. 2018;103(5):85764.

14. Silverberg MJ, Lau B, Achenbach CJ, Jing Y, Althoff KN, D'Souza G, et al. Cumulative Incidence of Cancer Among Persons With HIV in North America: A Cohort Study. Ann Intern Med. 2015;163(7):507-18.

15. Gopal S, Patel MR, Yanik EL, Cole SR, Achenbach CJ, Napravnik S, et al. Temporal trends in presentation and survival for HIV-associated lymphoma in the antiretroviral therapy era. J Natl Cancer Inst. 2013;105(16):1221-9.

16. Hleyhel M, Belot A, Bouvier AM, Tattevin P, Pacanowski J, Genet P, et al. Risk of AIDS-defining cancers among HIV-1-infected patients in France between 1992 and 2009: results from the FHDH-ANRS CO4 cohort. Clin Infect Dis. 2013;57(11):163847.

17. Mezger NCS, Feuchtner J, Griesel M, Hammerl L, Seraphin TP, Zietsman A, et al. Clinical presentation and diagnosis of adult patients with non-Hodgkin lymphoma in Sub-Saharan Africa. Br J Haematol. 2020;190(2):209-21.

18. Barta SK, Xue X, Wang D, Tamari R, Lee JY, Mounier N, et al. Treatment factors 
affecting outcomes in HIV-associated non-Hodgkin lymphomas: a pooled analysis of 1546 patients. Blood. 2013;122(19):3251-62.

19. Ehrenkranz P, Rosen S, Boulle A, Eaton JW, Ford N, Fox MP, et al. The revolving door of HIV care: Revising the service delivery cascade to achieve the UNAIDS 95-95-95 goals. PLoS Med. 2021;18(5):e1003651.

20. Boue F, Gabarre J, Gisselbrecht C, Reynes J, Cheret A, Bonnet F, et al. Phase II trial of CHOP plus rituximab in patients with HIV-associated non-Hodgkin's lymphoma. J Clin Oncol. 2006;24(25):4123-8.

21. Ribera JM, Oriol A, Morgades M, Gonzalez-Barca E, Miralles P, Lopez-Guillermo A, et al. Safety and efficacy of cyclophosphamide, adriamycin, vincristine, prednisone and rituximab in patients with human immunodeficiency virus-associated diffuse large B-cell lymphoma: results of a phase II trial. $\mathrm{Br} \mathrm{J}$ Haematol. 2008;140(4):411-9.

22. Sparano JA, Lee JY, Kaplan LD, Levine AM, Ramos JC, Ambinder RF, et al. Rituximab plus concurrent infusional EPOCH chemotherapy is highly effective in HIV-associated B-cell non-Hodgkin lymphoma. Blood. 2010;115(15):3008-16.

23. Little RF, Pittaluga S, Grant N, Steinberg SM, Kavlick MF, Mitsuya H, et al. Highly effective treatment of acquired immunodeficiency syndrome-related lymphoma with dose-adjusted EPOCH: impact of antiretroviral therapy suspension and tumor biology. Blood. 2003;101(12):4653-9.

24. Kimani S, Painschab MS, Kaimila B, Kasonkanji E, Zuze T, Tomoka T, et al. Safety and efficacy of rituximab in patients with diffuse large B-cell lymphoma in Malawi: a prospective, single-arm, non-randomised phase 1/2 clinical trial. Lancet Glob Health. 2021;9(7):e1008-16.

25. Kaplan LD, Lee JY, Ambinder RF, Sparano JA, Cesarman E, Chadburn A, et al. Rituximab does not improve clinical outcome in a randomized phase 3 trial of CHOP with or without rituximab in patients with HIV-associated non-Hodgkin lymphoma: 
AIDS-Malignancies Consortium Trial 010. Blood. 2005;106(5):1538-43.

26. Barta SK, Samuel MS, Xue X, Wang D, Lee JY, Mounier N, et al. Changes in the influence of lymphoma- and HIV-specific factors on outcomes in AIDS-related non-Hodgkin lymphoma. Ann Oncol. 2015;26(5):958-66.

27. Ruppert AS, Dixon JG, Salles G, Wall A, Cunningham D, Poeschel V, et al. International prognostic indices in diffuse large B-cell lymphoma: a comparison of IPI, R-IPI, and NCCN-IPI. Blood. 2020;135(23):2041-8.

28. Sehn LH, Salles G. Diffuse Large B-Cell Lymphoma. N Engl J Med. 2021;384(9):842-58. 


\section{Figure legend}

Fig. 1 Distribution of HIV-positive lymphoma

Abbreviations: DLBCL, diffuse large B-cell lymphoma; BL, Burkitt's lymphoma; PBL, plasmablastic lymphoma; ARL, NOS, AIDS-related lymphoma, not otherwise specified; HL, Hodgkin's lymphoma; NKTL, natural killer/T-cell lymphoma; ALCL, anaplastic large cell lymphoma; and SLL, small lymphocytic lymphoma.

Fig. 2 Kaplan-Meier survival curves for (A) 1-year $(\mathrm{N}=94)$ and (B) 2-year $(\mathrm{N}=65)$ progression-free survival (left) and overall survival (right) in 94 HIV/AIDS-associated NHL patients.

Fig. 3 (A) Kaplan-Meier survival curves for 1-year $(\mathrm{N}=87)$ progression-free survival (left) and overall survival (right) in 87 AIDS-related DLBCL patients. (B) ROC curve for one-year OS. Abbreviations: LDH, lactate dehydrogenase; ESR, erythrocyte sedimentation rate; AGE, age; LYM, lymphocyte; IPI, International Prognosis Index; CD4, CD4 T-cell count; CD4/CD8, CD4/CD8 ratio; RDW, red cell distribution width; and ALB, albumin.

Table 1 Baseline patient characteristics

Table 2 Univariate and multivariate analyses for 1-year PFS and OS of HIV/AIDS-associated NHL ( $=94)$

Table 3 Univariate and multivariate analyses for 1-year PFS and OS of AIDS-related $\operatorname{DLBCL}(\mathrm{N}=65)$

Supplementary Figure 1. Kaplan-Meier estimates for 1-year OS of the opportunistic infection outcomes of HIV-positive lymphomas.

Supplementary Figure 2. Survival curves of the (A) age, (B) CD4 T-cell count, (C) CD4/CD8 ratio, (D) LDH, (E) IPI, (F) ESR, (G) RDW ratio, (H) LYM, and (I) ALB stratifications for 1-year PFS (left) and OS (right) in HIV/AIDS-associated NHL $(\mathrm{N}=94)$. 
Supplementary Figure 3. Survival curves of the (A) age, (B) CD4 T-cell count, (C) CD4/CD8 ratio, (D) LDH, (E) IPI, (F) ESR, (G) RDW ratio, (H) LYM, and (I) ALB stratifications for 1-year PFS (left) and OS (right) in AIDS-related DLBCL (N=65).

Supplementary Table 1 Baseline infections

Supplementary Table 2 HIV treatments

Supplementary Table 3 Lymphoma treatments and outcomes 


\section{Figures}

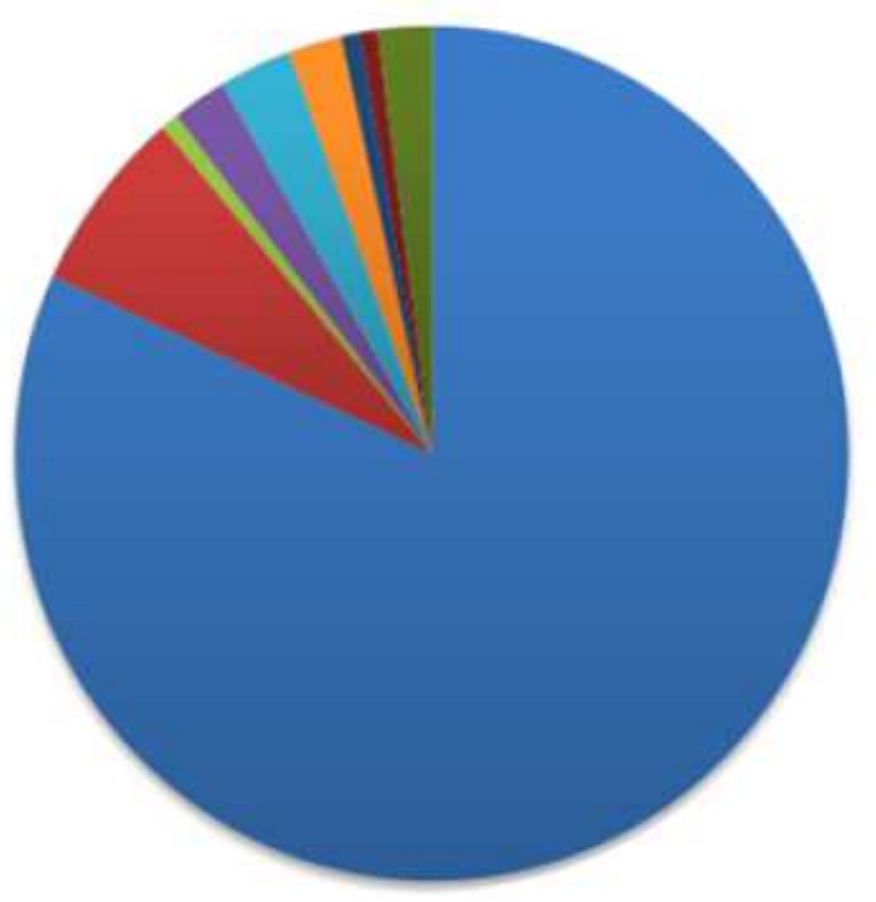

DLBCL $(81.8 \%, 117)$

BL $(7.0 \%, 10)$

PBL $(0.7 \%, 1)$

ARL, not further classified $(2.1 \%, 3)$

HL $(2.8 \%, 4)$

$\operatorname{NKTL}(2.1 \%, 3)$

$\operatorname{ALCL}(0.7 \%, 1)$

$\operatorname{SLL}(0.7 \%, 1)$

non-ARL $(2.1 \%, 3)$

Figure 1

Distribution of HIV positive lymphoma Abbreviations: DLBCL, diffuse large B cell lymphoma; BL, Burkitt's lymphoma; PBL, plasmablastic lymphoma; ARL, NOS, AIDS related lymphoma, not otherwise specified; HL, Hodgkin's lymphoma; NKTL, natural killer/T cell lymphoma; ALCL, anaplastic large cell lymphoma; and SLL, small lymphocytic lymphoma. 

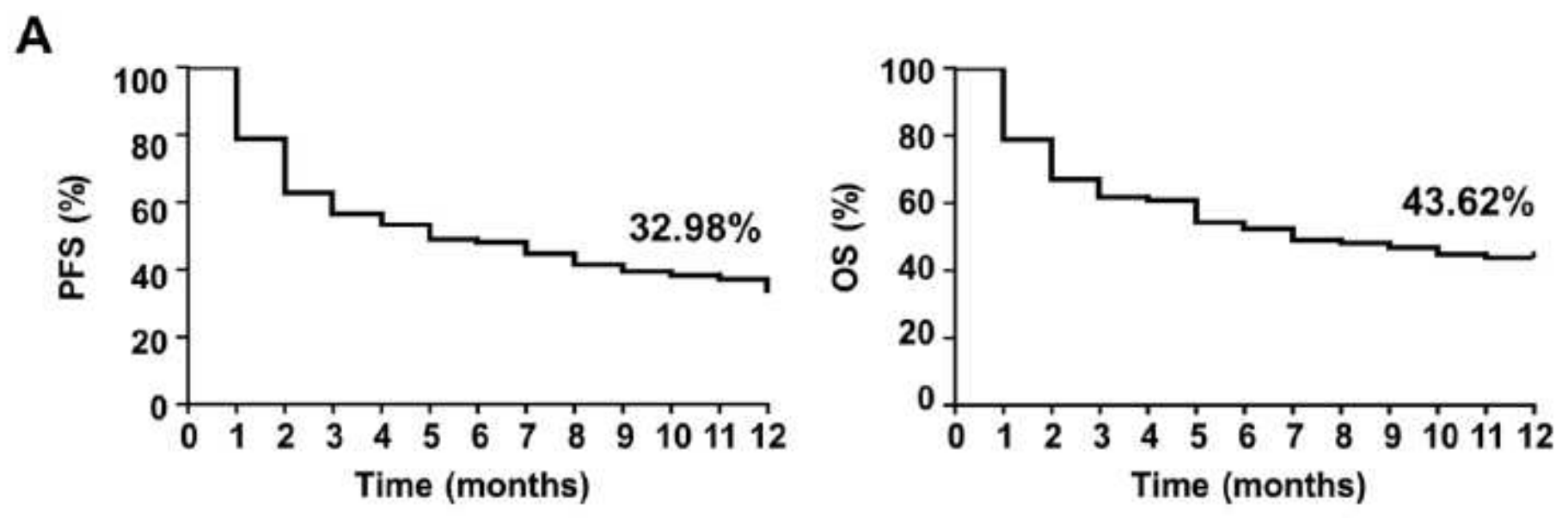

B
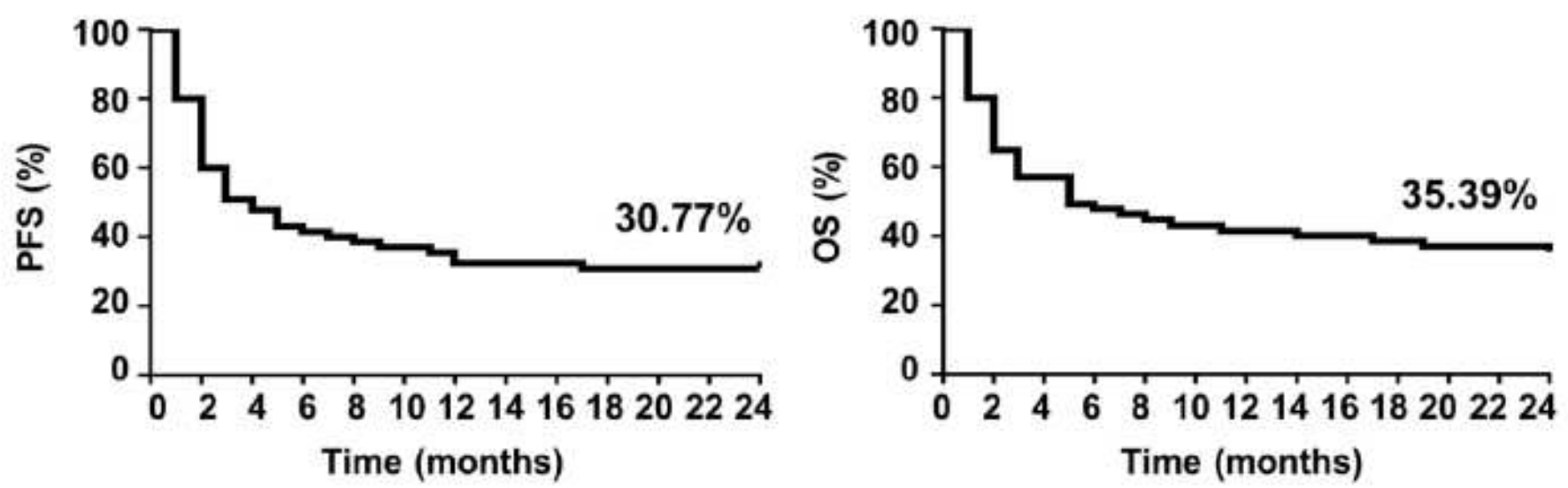

Figure 2

Kaplan Meier survival curves for $(A) 1$ year $(N=94)$ and $(B) 2$ year $(N=65)$ progression free survival (left) and overall survival (right) in 94 HIV/AIDS associated NHL patients.
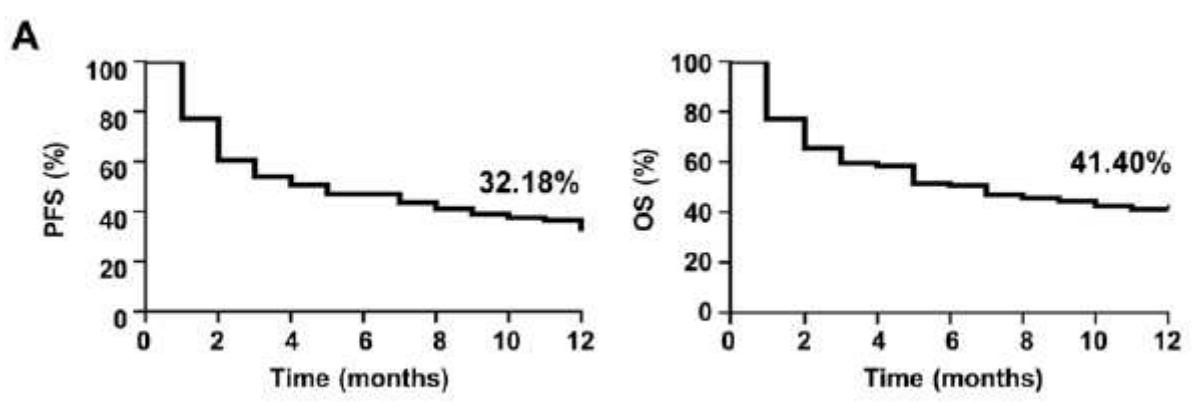

B

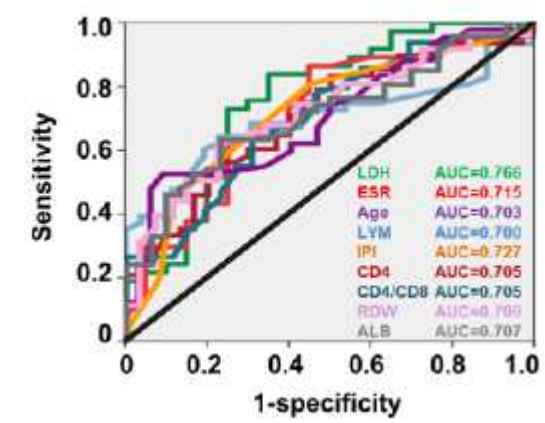

Figure 3

(A) Kaplan Meier survival curves for 1 year $(\mathrm{N}=87)$ progression free survival (left) and overall survival (rig ht) in 87 AIDS related DLBCL patients. (B) ROC curve for one year OS. Abbreviations: LDH, lactate dehydrogenase; ESR, erythrocyte sedimentation rate; AGE, age; LYM, lymphocyte; IPI, International 
Prognosis Index; CD4, CD4 T cell count; CD4/CD8, CD4/CD8 rat io; RDW, red cell distribution width; and ALB, albumin.

\section{Supplementary Files}

This is a list of supplementary files associated with this preprint. Click to download.

- table.pdf

- SupplementaryMaterial.pdf 